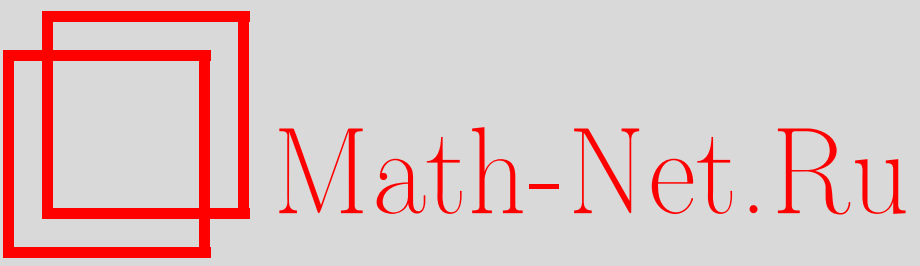

Н. Н. Ганиходжаев, У. А. Розиков, Описание периодических крайних гиббсовских мер некоторых решеточных моделей на дереве Кэли, ТМФ, 1997, том 111, номер 1, 109-117

DOI: https://doi.org/10.4213/tmf993

Использование Общероссийского математического портала Math-Net.Ru подразумевает, что вы прочитали и согласны с пользовательским соглашением

http: //www . mathnet.ru/rus/agreement

Параметры загрузки :

IP : 35.173 .219 .149

26 апреля 2023 г., 04:28:37 


\author{
ТЕОРЕТИЧЕСКАЯ \\ И МАТЕМАТИЧЕСКАЯ \\ ФИЗИКА \\ Том 111, № 1 \\ апрель, 1997
}

Н. Н. Ганиходжаев*, У. А. Розиков*

\title{
ОПИСАНИЕ ПЕРИОДИЧЕСКИХ КРАЙНИХ ГИББСОВСКИХ МЕР НЕКОТОРЫХ РЕШЕТОЧНЫХ МОДЕЛЕЙ НА ДЕРЕВЕ КЭЛИ
}

Доказаны единственность трансляционно-инвариантных гиббсовских мер антиферромагнитной модели Поттса с внешним полем и сушествование несчетного числа крайних гиббсовских мер для модели Изинга с внешним полем. Описаны классы нормальных делителей конечного индекса группового представления дерева Кэли. Для модели Изинга с нулевым внешним полем построены периодические гиббсовские меры, инвариантные относительно нормальных делителей индекса два; с помощью этих мер доказано существование несчетного числа крайних гиббсовских мер антиферромагнитной модели Изинга.

\section{ВВЕДЕНИЕ}

Дерево Кэли $\mathcal{T}^{k}$ (или решетка Бете по другой терминологии, см. [1]) порядка $k \geqslant 1$ есть бесконечное дерево, т.е. граф̆ без циклов, из каждой вершины которого выходят ровно $k+1$ ребер.

Отсутствие замкнутых контуров на дереве Кэли позволяет применить теорию марковских случайных полей на деревьях и рекуррентные уравнения этой теории, что дает возможность во многих случаях точно решить ряд решеточных моделей [2-9].

Цель настоящей работы состоит в том, чтобы для моделей на дереве Кэли:

1) доказать единственность трансляционно-инвариантных гиббсовских мер антиферромагнитной модели Поттса с внешним полем;

2) доказать существование несчетного числа крайних распределений Гиббса модели Изинга с внешним полем;

3) дать описание классов нормальных делителей конечного индекса группового представления дерева Кэли, что позволяет рассмотреть задачу описания периодических гиббсовских мер для моделей, определенных на дереве Кэли;

4) описать периодические гиббсовские меры модели Изинга;

5) описать новый класс крайних гиббсовских мер для антиферромагнитной модели Изинга с нулевым внешним полем.

\footnotetext{
${ }^{*}$ Институт математики Академии наук Республики Узбекистан
} 


\section{1. КОНСТРУКЦИЯ И ОПИСАНИЕ ПРЕДЕЛЬНЫХ ГИББСОВСКИХ МЕР НА ДЕРЕВЕ КЭЛИ}

Пусть $\mathcal{T}^{k}=(V, L)$ - решетка Бете порядка $k \geqslant 1$. Расстояние $d(x, y), x, y \in V$ на $\mathcal{T}^{k}$ определяется по формуле

$$
\begin{aligned}
d(x, y)= & \min \left\{d \mid x=x_{0}, x_{1}, \ldots, x_{d-1}, x_{d}=y \in V\right. \text { такие, что пары } \\
& \left.\left\langle x_{0}, x_{1}\right\rangle, \ldots,\left\langle x_{d-1}, x_{d}\right\rangle-\text { ближайшие соседи }\right\} .
\end{aligned}
$$

Последовательность $\pi=\left\{x=x_{0}, x_{1}, \ldots, x_{d-1}, x_{d}=y \in V\right\}$, реализуюшая указанньй минимум, называется путем из $x$ в $y$.

Для произвольной точки $x^{0} \in V$ положим

$$
\begin{aligned}
W_{n} & =\left\{x \in V \mid d\left(x, x^{0}\right)=n\right\}, \\
V_{n} & =\bigcup_{m=0}^{n} W_{m}=\left\{x \in V \mid d\left(x, x^{0}\right) \leqslant n\right\}, \\
L_{n} & =\left\{l=\langle x, y\rangle \in L: x, y \in V_{n}\right\} .
\end{aligned}
$$

Говорят, что $x<y$, если путь из $x^{0}$ в $y$ проходит через $x$. При этом вершина $y$ называется "прямым потомком" вершины $x$, если $y>x$ и $x, y$ являются ближайшими соседями. Если $S(x)$ - совокупность “прямых потомков" вершины $x$, то для любой вершины $x \in V$, отличной от $x^{0}$, имеем $|S(x)|=k$ и $\left|S\left(x^{0}\right)\right|=k+1[6]$.

Гамильтониан модели Изинга с внешним полем на дереве Кэли имеет вид

$$
H_{V_{n}}(\sigma)=-\mathcal{J} \sum_{\langle x, y\rangle \in L_{n}} \sigma(x) \sigma(y)-\alpha \sum_{x \in V_{n}} \sigma(x),
$$

где $\alpha, \mathcal{J} \in R, \sigma(x) \in\{-1,1\}, x \in V$.

Пусть $A \subset V$ - конечное подмножество. Обозначим через $\Omega_{A}=\{-1,1\}^{A}$ пространство конфигураций, определенных на множестве $A$. Пусть $h_{x} \in R$-вешественнозначная функция $x \in V$. Рассмотрим для каждого $n$ меру $\mu_{n}$ на $\Omega_{V_{n}}$, определенную формулой

$$
\mu_{n}\left(\sigma_{n}\right)=Z_{n}^{-1} \exp \left\{T^{-1} H_{V_{n}}(\sigma)+\sum_{x \in V_{n}} h_{x} \sigma(x)\right\}
$$

где $T>0, \sigma_{n}=\left\{\sigma(x): x \in V_{n}\right\} \in \Omega_{V_{n}}$ и $Z_{n}$ - нормируюший множитель. Условия согласования для $\mu_{n}\left(\sigma_{n}\right), n \geqslant 1$, определяются равенством

$$
\sum_{\sigma^{(n)}} \mu_{n}\left(\sigma_{n-1}, \sigma^{(n)}\right)=\mu_{n-1}\left(\sigma_{n-1}\right),
$$

где $\sigma^{(n)}=\left\{\sigma(x): x \in W_{n}\right\}$. В этом случае существует распределение Гиббса $\mu$ на $\Omega_{V}$. Оно является марковским и называется цепью Маркова со взаимодействиями $\mathcal{J}$ и $\alpha$. 
ТеОрема $1.1[7,8]$. Меры (1.2) удовлетворяют условию согласования (1.3) тогда и только тогда, когда для $\forall x \in V$

$$
h_{x}=\alpha / T+\sum_{y \in S(x)} \operatorname{arcth}\left(\theta \operatorname{th} h_{y}\right)
$$

əде $\theta=\operatorname{th}(\mathcal{J} / T)$.

В модели Поттса с внешним полем на дереве Кэли спиновые переменные $\sigma(x), x \in V$, принимают значения $\sigma_{1}, \sigma_{2}, \ldots, \sigma_{q}$ и гамильтониан имеет вид

$$
\widetilde{H}_{V_{n}}(\sigma)=-\mathcal{J} \sum_{\langle x, y\rangle \in L_{n}} \delta_{\sigma(x) \sigma(y)}-\alpha \sum_{x \in V_{n}} \delta_{\sigma(x) \sigma_{1}},
$$

где $\sigma$ - символ Кронекера. Антиферромагнитная модель Поттса определяется гамильтонианом (1.5) при $\mathcal{J}<0$.

Предположим, что $\sigma_{1}, \sigma_{2}, \ldots, \sigma_{q} \in R^{q-1}$ такие, что

$$
\sigma_{i} \sigma_{j}= \begin{cases}1, & \text { если } i=j, \\ -1 / q-1, & \text { если } i \neq j .\end{cases}
$$

Тогда для любых $x, y \in V$ находим

$$
\frac{q-1}{q}\left(\sigma(x) \sigma(y)+\frac{1}{q-1}\right)=\delta_{\sigma(x) \sigma(y)},
$$

откуда $\widetilde{H}_{V_{n}}(\sigma)$ имеет вид

$$
\widetilde{H}_{V_{n}}(\sigma)=-\mathcal{J} \sum_{\langle x, y\rangle \in L_{n}} \sigma(x) \sigma(y)-\alpha \sum_{x \in V_{n}} \sigma(x) \sigma_{1} .
$$

Пусть $A \subset V$ - конечное подмножество. Обозначим через $\Omega_{A}=\left\{\sigma_{1}, \ldots, \sigma_{q}\right\}^{A}$ пространство конфигураций на множестве $A$. Конечномерное распределение меры $\mu$ ( $\mu-$ распределение Гиббса) в объеме $V_{n}$ определяется формулой

$$
\mu_{n}\left(\sigma_{n}\right)=\widetilde{Z}_{n}^{-1} \exp \left\{T^{-1} \widetilde{H}_{V_{n}}(\sigma)+\sum_{x \in W_{n}} \tilde{h}_{x} \sigma(x)\right\},
$$

где $\widetilde{Z}_{n}$ - нормируюший множитель, а $\tilde{h}_{x} \in R^{q-1}$.

ТеОрема $1.2[3,4]$. Меръ (1.8) удовлетворяют (1.3) тогда и только тогда, когда для $\forall x \in V$

$$
\tilde{h}_{x}=\sum_{y \in S(x)} F\left(\tilde{h}_{y}, \theta_{1}, \alpha\right)
$$

здесь $F$ - преобразование, переводящее $R^{q-1}$ в себя по следующей формуле:

$$
h_{i}^{\prime}=\frac{\alpha}{T} \delta_{1 i}+\ln \left[\frac{\left(\theta_{1}-1\right) \exp h_{i}+\sum_{j=1}^{q-1} \exp h_{j}+1}{\sum_{j=1}^{q-1} \exp h_{j}+\theta_{1}}\right], \quad i=\overline{1, q-1},
$$

где $\theta_{1}=\exp (\mathcal{J} / T)$, а $h=\left(h_{1}, h_{2}, \ldots, h_{q-1}\right) \in R^{q-1}$. Каждой совокупности векторов $\left\{h_{x}, x \in V\right\}$, удовлетворяющих (1.9), соответствует единственная гиббсовская мера $\mu$ на $\Omega_{V}$. 


\section{2. ТРАНСЛЯЦИОННО-ИНВАРИАНТНЫЕ ГИББСОВСКИЕ МЕРЫ АНТИФЕРРОМАГНИТНОЙ МОДЕЛИ ПОТТСА С ВНЕШНИМ ПОЛЕМ}

В этом разделе мы приводим конструкцию гиббсовских мер, инвариантных относительно всех пространственных сдвигов решетки $\mathcal{T}^{k}$. В [3, 4] для ферромагнитной модели Поттса с нулевым полем доказано сушествование $q$ трансляционно-инвариантных и несчетное число не трансляционно-инвариантных гиббсовских мер и также дано их конструктивное описание.

Предположим, что $\tilde{h}_{x}=h=\left(h_{1}, h_{2}, \ldots, h_{q-1}\right)$ для $\forall x \in V$. Тогда из теоремы $1.2 \mathrm{c}$ помошью замены $z_{i}=\exp h_{i}$ получим

$$
z_{i}=\exp \left(\frac{\alpha}{T} \delta_{1 i}\right)\left[\frac{\left(\theta_{1}-1\right) z_{i}+\sum_{j=1}^{q-1} z_{j}+1}{\sum_{j=1}^{q-1} z_{j}+\theta_{1}}\right]^{k}, \quad i=\overline{1, q-1}
$$

Заметим, что при $0<\theta_{1}<1(\mathcal{J}<0)$ система $(2.1)$ имеет единственное решение $z^{*}=\left(z_{*}, 1,1, \ldots, 1\right)$. Обозначим через $\mu_{*}$ гиббсовскую меру, соответствуюшую совокупности векторов $\left\{h_{x}=\left(\ln z_{*}, 0,0, \ldots, 0\right) \forall x \in V\right\}$.

Таким образом, мы доказали следуюшую теорему.

Теорема 2.2. При $q>1, \quad k \geqslant 1, \mathcal{J}<0, \alpha \in R$ в модели Поттса трансляиионно-инвариантная гиббсовская мера единственна и эта мера есть $\mu_{*}$.

\section{3. КРАЙНИЕ ГИББСОВСКИЕ МЕРЫ МОДЕЛИ ИЗИНГА С ВНЕШНИМ ПОЛЕМ}

Для того чтобы мера была трансляционно-инвариантной для модели (1.1), необходимо выполнение условия $h_{x}=h \in R \forall x \in V$. Тогда из (1.4) получим

$$
h=\frac{\alpha}{T}+k \operatorname{arcth}(\theta \operatorname{th} h) .
$$

Из свойств функции $\frac{\alpha}{T}+k \operatorname{arcth}(\theta \operatorname{th} h)$ следует, что при $\alpha \in(-(k-1) \mathcal{J},(k-1) \mathcal{J})$, $\mathcal{J}>0$, уравнение (3.1) имеет два устойчивых решения $h_{*}^{(1)}<h_{*}^{(2)}$ и одно неустойчивое решение $h_{*}^{(3)}$, лежашее между $h_{*}^{(1)}$ и $h_{*}^{(2)}$.

Рассмотрим совокупности величин $\left\{h_{x}=h_{*}^{(1)} \forall x \in V\right\}$ и $\left\{h_{x}=h_{*}^{(2)} \forall x \in V\right\}$ и обозначим через $\mu^{(1)}$ и $\mu^{(2)}$ соответствуюшие им распределения Гиббса.

Теорема 3.1 [5,9]. Меры $\mu^{(1)}$ и $\mu^{(2)}$ являются крайними распределениями Гиб$6 c a$.

Произвольному пути $\pi$, конечному или бесконечному, стандартным образом можно сопоставить число $t \in[0,1]$ (см. [2-4]). Нетрудно показать, что для любого $t \in[0,1]$ однозначно определяется совокупность величин $h^{\pi(t)}=\left\{h_{x}^{\pi(t)}=h_{x}^{\pi(t)}\left(h_{*}^{(1)}, h_{*}^{(2)}\right)\right.$, $x \in V\}$, которая удовлетворяет (1.4).

Обозначим через $\mu^{t}$ гиббсовскую меру, соответствуюшую $h^{\pi(t)}$. 
ТЕОРема 3.2. Для любого $t \in[0,1]$ гиббсовская мера $\mu^{t}$ является крайней.

ДоКАЗАТЕЛЬСТво аналогично доказательству теоремы 3.2 работы [2].

\section{4. ГРУППОВОЕ ПРЕДСТАВЛЕНИЕ И АВТОМОРФИЗМЫ ДЕРЕВА КЭЛИ}

Пусть $G_{k+1}-$ свободное произведение $k+1$ циклических групп второго порядка с образуюшими $a_{1}, a_{2}, \ldots, a_{k+1}$, соответственно.

ПРЕДЛОЖЕНИЕ 4.1 [10]. Существует взаимно однозначное соответствие между множеством вершин $V$ дерева Кәли порядка $k$ и группой $G_{k+1}$.

Рассмотрим на группе $G_{k+1}$ преобразования левого (правого) сдвигов, определяемых следующим образом. Для $g_{0} \in G_{k+1}$ положим

$$
T_{g_{0}}(h)=g_{0} h \quad\left(T_{g_{0}}(h)=h g_{0}\right) \quad \forall h \in G_{k+1}
$$

Совокупность всех левых (правых) сдвигов на $G_{k+1}$ изоморфна группе $G_{k+1}$.

В соответствии с предложением 4.1 любое преобразование $S$ групшы $G_{k+1}$ индуцирует преобразование $\widehat{S}$ на множестве вершин $V$ дерева Кэли. Очевидна следующая теорема.

Теорема 4.1. Группа левых (правых) сдвигов на правом (левом) представлении дерева Кәли является группой транслячий дерева Кәли.

\section{5. НОРМАЛЬНЫЕ ДЕЛИТЕЛИ КОНЕЧНОГО ИНДЕКСА ГРУППОВОГО ПРЕДСТАВЛЕНИЯ ДЕРЕВА КЭЛИ}

Любой элемент $x \in G_{k+1}$ имеет следуюший вид:

$$
x=a_{i_{1}} a_{i_{2}} \ldots a_{i_{n}}, \quad \text { где } 1 \leqslant i_{m} \leqslant k+1, \quad m=\overline{1, n} .
$$

Число $n$ называется длиной слова $x$ и обозначается через $l(x)$. Число букв $a_{i}, i=$ $\overline{1, k+1}$, участвуюших в несократимой записи слова $x$, обозначим через $\omega_{x}\left(a_{i}\right)$.

ПРЕДЛОЖЕНИЕ 5.1 (см. [11,12]). Если $\varphi-$ гомоморфизм группы $G_{k+1}$ с ядром $H$, то $H$ является нормальным делителем группь $G_{k+1} u \varphi\left(G_{k+1}\right) \simeq G_{k+1}: H$ $\left(G_{k+1}: H\right.$ - фактор-группа $)$, т.е. индекс $\left|G_{k+1}: H\right|$ совпадает с порядком $\left|\varphi\left(G_{k+1}\right)\right|$ zрynnbl $\varphi\left(G_{k+1}\right)$.

Вследствие предложения 5.1 для построения нормального делителя конечного индекса группы $G_{k+1}$ необходимо построить гомоморфизм группы $G_{k+1}$ в некоторую конечную группу. 
ОПРЕДЕЛЕНИЕ 5.1. Пусть $M_{1}, M_{2}, \ldots, M_{m}$ - некоторые множества и $M_{i} \neq M_{j}$ при $i \neq j \quad(i, j=\overline{1, m})$. Пересечение $\bigcap_{i=1}^{m} M_{i}$ назовем сократимым, если сушествует $i_{0}$ $\left(1 \leqslant i_{0} \leqslant m\right)$ такое, что

$$
\bigcap_{i=1}^{m} M_{i}=\left(\bigcap_{i=1}^{i_{0}-1} M_{i}\right) \cap\left(\bigcap_{i=i_{0}+1}^{m} M_{i}\right) .
$$

Пусть $N_{k}=\{1,2, \ldots, k+1\}$.

Teорема 5.1. Для любого $\varnothing \neq A \subseteq N_{k}$ существует подгруппа $H_{A} \subset G_{k+1}$ co следующими свойствами:

а) $H_{A}-$ нормальный делитель и $\left|G_{k+1}: H_{A}\right|=2$;

б) $H_{A} \neq H_{B} \partial \Omega_{я} \forall A \neq B \subseteq N_{k}$;

в) $\left|H_{A} \cap H_{B}\right|=\infty u H_{A} \cap H_{B} \subset H_{A \triangle B}, A, B \subseteq N_{k}$;

г) если $A_{1}, A_{2}, \ldots, A_{m} \subseteq N_{k}$ и $A_{i} \cap A_{j}=\varnothing$ для любых $i \neq j=\overline{1, m}$, mо

$$
\bigcap_{i=1}^{m} H_{A_{i}} \subset H_{\bigcup_{i=1}^{m} A_{i}}
$$

д) пусть $A_{1}, A_{2}, \ldots, A_{m} \subseteq N_{k}$. Если $\bigcap_{i=1}^{m} H_{A_{i}}$ - несократимое пересечение, то оно является нормальным делителем индекса $2^{m}$;

е) для любого $m=1,2 k$ (где $k$ - порядок решетки) существуют несократимые пересечения

$$
H_{m}=\bigcap_{i=1}^{m} H_{A_{i}}
$$

ДокаЗАТЕЛЬСтво. Пусть $\varnothing \neq A \subseteq N_{k}$. Определим отображение

$$
f_{A}(x)=\left\{\begin{array}{lll}
1, & \text { если } \sum_{i \in A} \omega_{x}\left(a_{i}\right) \text { четно, } \\
-1, & \text { если } \sum_{i \in A} \omega_{x}\left(a_{i}\right) \text { нечетно. }
\end{array}\right.
$$

Легко заметить, что $f_{A}$ является гомоморфизмом, т.е. для любых $x, y \in G_{k+1}$ справедливо равенство $f_{A}(x y)=f_{A}(x) f_{A}(y)$. В силу предложения 5.1 $H_{A}=\left\{x \in G_{k+1}\right.$ : $\sum_{i \in A} \omega_{x}\left(a_{i}\right)$ четно $\}$ является нормальным делителем индекса 2 . Нетрудно убедиться, что $H_{A}$ удовлетворяет всем свойствам теоремы 5.1. Теорема доказана.

Пусть $x=a_{i_{1}} a_{i_{2}} \ldots a_{i_{n}}$, где $1 \leqslant i_{m} \leqslant k+1, m=\overline{1, n}$. Для $x \in\left\{y \in G_{k+1}: l(y)=n\right\}$ положим $\nu_{x}\left(a_{j}\right)=\left\{m \in N_{n-1}: i_{m}=j\right\}$. Например, если $x=a_{1} a_{4} a_{8} a_{4} a_{1} a_{2} a_{4}$, то $\nu_{x}\left(a_{4}\right)=\{2,4,7\}$. Обозначим через $\alpha(x)$ число образуюших элемента $x$.

Теорема 5.2. Пусть $е \neq x_{0} \in G_{k+1}$. Тогда существует нормальный делитель $H_{x_{0}}$ конечного индекса, не содержащий әлемента $x_{0}$, такой, что

$$
\alpha\left(x_{0}\right)+1 \leqslant\left|G_{k+1}: H_{x_{0}}\right| \leqslant\left(l\left(x_{0}\right)+1\right) !
$$


ДокАЗАТЕльСтво. Пусть $\alpha\left(x_{0}\right)=m$. Обозначим через $a_{1}^{\prime}, a_{2}^{\prime}, \ldots, a_{m}^{\prime}, 1 \leqslant m \leqslant$ $k+1$, образуюшие $a_{i}, i=\overline{1, k+1}$, в несократимой записи элемента $x$. Тогда $x_{0}$ имеет вид

$$
x_{0}=a_{i_{1}}^{\prime} a_{i_{2}}^{\prime} \ldots a_{i_{s}}^{\prime}, \quad \text { где } 1 \leqslant i_{s} \leqslant k+1, \quad s=\overline{1, n} .
$$

В симметрической группе $S_{n+1}$, переставляюшей символы $1,2, \ldots, n+1$, выберем подстановки $\pi_{i}, i=\overline{1, m}$, следуюшим образом: если $\nu_{x_{0}}\left(a_{j}\right)=\left\{j_{1}, j_{2}, \ldots, j_{m_{1}}\right\}$, где $j_{k_{1}} \in N_{n-1}$ и $k_{1}=\overline{1, m_{1}}$, то

$$
\pi_{j}=\left(\begin{array}{cccc}
1 \ldots j_{1} & j_{1}+1 \ldots j_{2} & j_{2}+1 \ldots j_{m_{1}} & j_{m_{1}}+1 \ldots n+1 \\
1 \ldots j_{1}+1 & j_{1} \ldots j_{2}+1 & j_{2} \ldots j_{m_{1}}+1 & j_{m_{1}} \ldots n+1
\end{array}\right), \quad j=\overline{1, m}
$$

Легко видеть, что $\pi_{j}^{2}=\pi_{0}, j=\overline{1, m}$, где $\pi_{0}$ - тождественная перестановка. Определим отображения $u:\left\{a_{1}, \ldots, a_{k+1}\right\} \rightarrow\left\{\pi_{1}, \ldots, \pi_{m}\right\}$ и $f_{x_{0}}: G_{k+1} \rightarrow S_{n+1}$ следуюшим образом:

$$
\begin{gathered}
u(x)=\left\{\begin{array}{lll}
\pi_{0}, & \text { если } x \neq a_{j}^{\prime}, \\
\pi_{j}, & \text { если } x=a_{j}^{\prime},
\end{array} \quad j=\overline{1, m},\right. \\
f_{x_{0}}(x)=f_{x_{0}}\left(a_{i_{1}} a_{i_{2}} \ldots a_{i_{s}}\right)=u\left(a_{i_{1}}\right) u\left(a_{i_{2}}\right) \ldots u\left(a_{i_{s}}\right) .
\end{gathered}
$$

Поскольку $\pi_{j}^{2}=\pi_{0}$, легко видеть, что $f_{x_{0}}$ является гомоморфиимом. Ядро этого гомоморфизма $H_{x_{0}}$ - нормальный делитель конечного индекса. Очевидно, что элемент $x$ лежит вне этого нормального делителя. Далее в силу предложения $5.1\left|G_{k+1}: H_{x_{0}}\right|=$ $\left|f_{x_{0}}\left(G_{k+1}\right)\right|$ и из построения $f_{x_{0}}$ видно, что

$$
\alpha\left(x_{0}\right)+1 \leqslant\left|G_{k+1}: H_{x_{0}}\right| \leqslant\left(l\left(x_{0}\right)+1\right) !
$$

Теорема доказана.

ПРЕДЛОЖЕНИЕ 5.3. Имеют место следующие равенства: 1) $H_{a_{i}}=H_{(i)}, \quad i=$ $1, k+1 ; 2)$ если $\alpha(x) \geqslant 2$, то $\left.\left|G_{k+1}: H_{x}\right|>3 ; 3\right)\left|G_{k+1}: H_{a_{i} a_{j}}\right|=6$ для $\forall i \neq j \in N_{k}$.

ДокАЗАТЕЛЬСТво следует из теорем 5.1 и 5.2 .

\section{6. ПЕРИОДИЧЕСКИЕ ГИББСОВСКИЕ МЕРЫ МОДЕЛИ ИЗИНГА НА ДЕРЕВЕ КЭЛИ}

Обычным образом вводятся понятия периодических гиббсовских мер, гамильтониана, конфигурашии и т.д. (см. $[13,14])$. Рассмотрим модель Изинга с нулевым полем, т.е. $\alpha=0$. Пусть $G_{k+1} / \widehat{G}_{*}=\left\{\widehat{G}_{*}, \widehat{G}_{* 1}\right\}-$ фактор-группа, где

$$
\widetilde{G}_{*}=H_{N_{k}}=\left\{x \in G_{k+1}: l(x) \text { четно }\right\}
$$




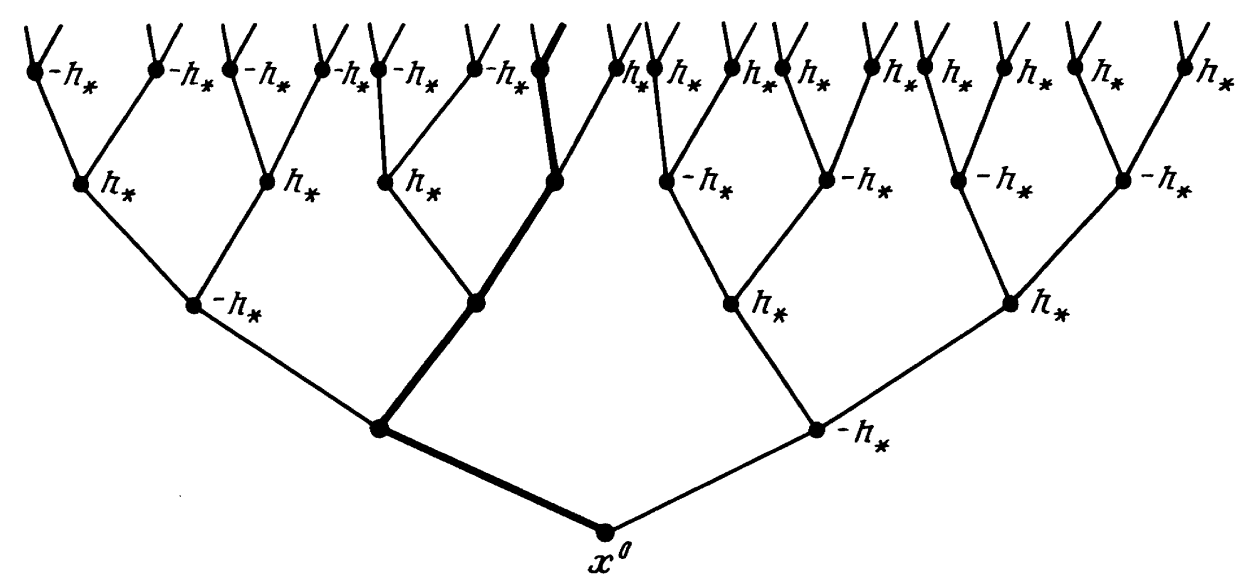

(см. раздел 5). Построим $\widehat{G}_{*}$-периодические распределения Гиббса. Для того чтобы получить периодические меры, положим в (1.4)

$$
h_{x}=\left\{\begin{array}{l}
h_{1}, \text { если } x \in \widehat{G}_{*}, \\
h_{2}, \text { если } x \in \widehat{G}_{* 1} .
\end{array}\right.
$$

Тогда получим

$$
\left\{\begin{array}{l}
h_{1}=k f\left(h_{2}, \theta\right), \\
h_{2}=k f\left(h_{1}, \theta\right),
\end{array}\right.
$$

где $\theta=\operatorname{th}(\mathcal{J} / T),-1<\theta<1, f(x, \theta)=\operatorname{arcth}(\theta \operatorname{th} x)$.

Легко заметить, что система уравнений имеет единственное решение $h_{*}=(0,0)$, если $-k^{-1} \leqslant \theta \leqslant k^{-1}$, и три решения $h_{*}^{(1)}=\left(-h_{*},-h_{*}\right), h_{*}^{(2)}=(0,0)$ и $h_{*}^{(3)}=\left(h_{*}, h_{*}\right)$ $\left(h_{*}>0\right)$, если $k^{-1} \leqslant \theta \leqslant 1$, и три решения $h_{*}^{\mp}=\left(h_{*},-h_{*}\right), h_{*}^{0}=(0,0), h_{*}^{ \pm}=\left(-h_{*}, h_{*}\right)$, если $-1<\theta<-k^{-1}$.

Теорема 6.1. Для ферромагнитной $(\mathcal{J}>0)$ (антиферромагнитной $(\mathcal{J}<0)$ ) модели Изинга существуют три (два) крайних периодических распределения Гиб$6 c a \hat{\mu}^{(1)}, \hat{\mu}^{(2)}, \hat{\mu}_{*}\left(\hat{\mu}^{\mp}, \hat{\mu}^{ \pm}\right)$.

ДокАЗАТЕЛЬСТво аналогично доказательству теоремы 3.2 работы [4].

Используя меры $\hat{\mu}^{\mp}, \hat{\mu}^{ \pm}$, построим несчетное число крайних распределений Гиббса. Можно доказать (ср. [2-4]), что при $-1<\theta<-k^{-1}$ совокупности величин

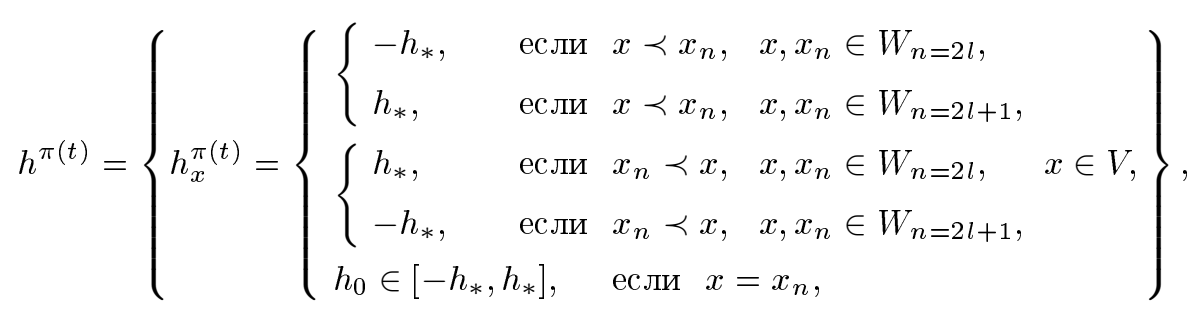


где $n=0,1,2, \ldots$ (см. рисунок), удовлетворяют (1.4). Эти совокупности величин различны при разных $t \in[0,1]$.

Обозначим через $\mu^{t}$ распределение Гиббса, соответствуюшее совокупности $h^{\pi(t)}$. Справедлива следующая теорема.

Теорема 6.2. Для любого $t \in[0,1]$ распределение Гиббса $\mu^{t}$ является крайним.

\section{Список литературы}

[1] Р. Бәкстер. Точно решаемые модели в статистической механике. М.: Мир, 1985.

[2] П. М. Блехер, Н. Н. Ганиходжаев. Теория вероятн. и ее примен. 1990. Т. 35. Вып. 2. C. $920-930$.

[3] Н. Н. Ганиходжаев. ДАН РУ. 1992. № 6-7. С. 4-7.

[4] Н. Н. Ганиходжаев. ТМФ. 1990. Т. 85. № 2. С. 163-175.

[5] К. Престон. Гиббсовские состояния на счетных множествах. М.: Мир, 1977.

[6] P. M. Bleher. Commun. Math. Phys. 1990. V. 128. P. 411-419.

[7] H. O. Georgii. Gibbs measures and phase trasitions (De Gru. studies in math.). Berlin, New York: de Gru., 1988.

[8] F. Spitzer. Ann. Prob. 1975. V. 3. P. 387-398.

[9] Y. Higuchi. Publ. RIMS Kyoto Univ. 1977. V. 3. P. 335-348.

[10] Н. Н. Ганиходжаев. ДАН РУз. 1994. № 5. С. 3-6.

[11] А. Г. Курош. Теория групп. М.: Наука, 1967.

[12] М.И. Каргаполов, Ю.И. Мерзляков. Основы теории групп. М.: Наука, 1982.

[13] С. А. Пирогов, Я. Г. Синай. ТМФ. 1975. Т. 25. № 3. С. 358-369; 1976. Т. 26. № 1. С. 61-76.

[14] Я. Г. Синай. Теория фазовых переходов. М.: Наука, 1980.

Поступила в редакцию $16 . \mathrm{IV} .1996$ г.

\section{N. N. Ganikhodzhaev, U. A. Rozikov \\ DISCRIPTION OF PERIODIC EXTREME GIBBS MEASURES OF SOME LATTICE MODELS ON THE CAYLEY TREE}

It is proved that the translational invariant extreme Gibbs measure is unique for the antiferromagnetic Potts model with the external field. The existence of uncountable numbers of the extreme Gibbs measures for the Ising model with the external field on the Cayley tree is proved. The classes of normal subgroups of finite index of group representation of the Cayley tree is constructed. The periodic extreme Gibbs measures invariant with respect to subgroups of index two for the Ising model are constructed and the existence of uncountable numberes of the nonperiodic extreme Gibbs measures for the antiferromagnetic Ising model is proved. 